\title{
BMJ Open Quality Reducing DNACPR complaints to zero: designing and implementing a treatment escalation plan using quality improvement methodology
}

Elizabeth Shermon, Laura Munglani, Sarah Oram, Linda William, Julian Abel

To cite: Shermon E, Munglani L, Oram S, et al. Reducing DNACPR complaints to zero: designing and implementing a treatment escalation plan using quality improvement methodology.BMJ Open Quality 2017;6:e00011. doi:10.1136/ bmjoq-2017-000011

Received 19 January 2017 Accepted 3 August 2017

\section{CrossMark}

Department of Palliative Care, Weston General Hospital, Weston-super-Mare, UK

Correspondence to Dr Elizabeth Shermon eshermon@nhs.net

\section{ABSTRACT}

Introduction Do Not Attempt Resuscitation (DNAR) decisions have traditionally formed the basis of ceiling of care discussions. However, poor quality discussions can lead to high patient and relative dissatisfaction, generating hospital complaints. Treatment escalation plans (TEPs) aim to highlight the wider remit of treatment options with a focus on effective communication. We aimed to improve TEP discussions and documentation at Weston General Hospital by introducing a standardised form.

Aims and method We aimed to develop a TEP document to reduce resuscitation-related complaints by improving communication and documentation. Qualitative and quantitative data were collected over 2 years and used to develop plan-do-study-act (PDSA) cycles using quality improvement methodology. Main barriers to improvement included time constraints and clinician's resistance. Results Analysis of patient liaison services data showed a progressive reduction in complaints regarding resuscitation, with no complaints having been received for the final six months of the project. Through use of a standardised form including treatment prompts, the quality of discussions and plans improved. Qualitative feedback demonstrated increased patient and relative satisfaction. In addition, junior doctors report the plans are helpful when making out-of-hours decisions.

Conclusion Development of a user-friendly form to document patient-guided TEPs helped junior doctors to lead advanced care planning discussions. The use of PDSA cycles demonstrated improvement in the quality of forms, which in turn improved communication, documentation and satisfaction. Future developments could include involvement of specialist teams to ensure TEP forms remain relevant to all clinical areas. In addition, with widespread use of the TEP forms, the traditional tick-box DNAR could be replaced to focus on patient-led care planning.

\section{PROBLEM}

Ambition 1 of the End of Life Ambitions Framework 2015-2020 is that each person is seen as an individual. ${ }^{1}$ Within this, the first building block is honest conversations: 'Everybody should have the opportunity for honest and well informed conversations about dying, death and bereavement.' Long-standing problems exist with both Do Not Attempt
Cardio-Pulmonary Resuscitation (DNACPR) discussions and documentation. Hospitals suffer repeated complaints, with poor recollection of discussions and poor documentation. The long-term outcomes of bereavement are significant, with increased morbidity, mortality, suicide and depression. DNACPR complaints are part of the picture of severe bereavement reactions. ${ }^{2}$ Experience of one of the authors (JA) in investigating complaints about DNACPR is one of trauma for those involved, with longstanding issues remaining unresolved for many months and poor understanding of the role of DNACPR among executive level staff, with inappropriate referral of senior doctors to the General Medical Council for investigation. Current DNACPR documentation has the hallmarks of a top-down approach to change with lack of quality improvement methodology in the design and implementation of both forms and guidelines. Documentation tends to be tick box in nature and does not enable a good process of discussion.

In the light of the above problems, we developed a quality improvement project with the main outcome being to reduce DNACPR complaints. Other primary outcome measures were to design a form that enabled good quality conversations, have good feedback from patients, relatives and the doctors who used them and to have an effective handover document in the out-of-hours setting.

Our quality improvement project was undertaken at a small district general hospital in southwest England, where the age of the local population is above the national average. The project was undertaken by four Foundation Year 1 doctors under the supervision of a palliative care consultant.

Previous junior doctors had highlighted problems with inadequate plans for treatment escalation, poor documentation about ceilings of care and unsatisfactory communication with patient and families. 
These factors were reflected in the numbers of DNACPR-related complaints and feedback from doctors that they felt ill-equipped in making treatment escalation decisions out of hours with unfamiliar patients.

A lack of a clear structure for resuscitation conversations and sporadic documentation regarding care plans was adversely affecting out-of-hours staff when trying to care for deteriorating patients, without prior knowledge of their wishes or concerns. In turn this resulted in distressing and unnecessary investigations and management for patients.

We set out to develop a user-friendly form to guide and document treatment escalation discussions and plans encompassing all elements of medical care. Through this we hoped to inspire doctors to hold such conversations with patients and their relatives in order to identify and understand their wishes, priorities and concerns. In addition, with evidence to suggest that advance planning helps to reduce inappropriate out-of-hours investigations, ${ }^{2}$ we hoped that through uniform documentation and filing of such discussions we could improve out-of-hours care of deteriorating patients by equipping doctors with an understanding of their agreed ceiling of care.

\section{BACKGROUND}

DNACPR decisions formed the main basis for treatment escalation decisions. However, these forms do not encompass the breadth of medical treatments available to patients and do not encourage good communication with patients or their families.

Evidence suggests that DNACPR decisions alone have the potential to be misinterpreted and result in the withholding of other medical treatment options which may be appropriate for and desired by patients. ${ }^{3}$ They also have the potential to be viewed as negative by patients as they focus on treatment being withheld and do not use the opportunity to explore what other treatment modalities would continue to be offered. ${ }^{4}$ Therefore, the current DNACPR process does not provide a holistic and positive approach to advanced care planning.

Furthermore, evidence currently suggests that communication with patients about DNACPR and treatment escalation decisions is poor and doctors often feel uncomfortable or ill-equipped to hold such discussions. ${ }^{4-8}$ This is often incongruent with patients' wishes, many of whom want to be involved with discussions about their ceiling of care and would welcome the opportunity to explore such matters. ${ }^{7}$ In addition, contrary to the beliefs held by some doctors, evidence suggests that patients rarely find being given information about their ceiling of care upsetting or distressing. ${ }^{9}$ This highlights a further problem with the current DNACPR process, in that the current design of the form does not encourage or support discussion with patients and families, which they would welcome.

Finally, since the current DNACPR form only documents decisions relating to Cardio-Pulmonary Resuscitation (CPR), where treatment escalation discussions are held with patients or their family, it is often difficult to locate this information in patient notes. Since individualised advance care planning should be encouraged, it is vital that this is documented in a way that is readily accessible and communicated effectively among all healthcare professionals. ${ }^{10} 11$ This is particularly beneficial out of hours, where doctors may be reviewing patients with whom they may be unfamiliar. Treatment escalation plans (TEPs) have been found to be helpful in prompting discussion with patients and relatives and providing an opportunity to explore their wishes. ${ }^{3}$ Previous TEP projects have demonstrated that TEP forms improve documentation about treatment escalation decisions and decrease uncertainty about ceilings of care. ${ }^{3}$

Unsurprisingly, these factors have contributed to TEPs being popular with junior doctors reducing anxiety and improving decision-making about deteriorating patients, especially out of hours. ${ }^{411}$

\section{Baseline measurement}

The main emphasis of the baseline measurement of the TEP forms was to monitor the output and aim for areas of further improvement. Once the initial baseline measurement was obtained, the collected data were reviewed and analysed, with a view to developing theories to be tested in plan-do-study-act (PDSA) cycles (see figure 1).

We used DNACPR-related complaints as our primary outcome measure. The previous Foundation Year 1 doctors had collected data and patient feedback, which we compared with our baseline results. DNACPR complaints related mainly $(83 \%)$ to poor communication: lack of communication between the patients/relatives regarding the DNACPR, families not being informed of decisions made by the medical team, incorrect information regarding comorbidities being documented and discharge issues such as a copy of the DNACPR not being sent home with the patients or to their general practitioner.

\section{DESIGN}

The initial TEP form used a three-step process, which consisted of a medical plan, but no ceiling of care or space for documentation.

Using the PDSA Quality Improvement methodology, the initial TEP forms were modified and moulded into a new two-paged form. The concepts of the forms were discussed with multiple specialties to accommodate the various levels of ceiling of care, that is, medical, surgicaland Intensive Care.

The TEP form consisted of three sections:

1. The initial decision made by the medical team centred on the patient's clinical condition. This decision was made by the most senior doctor to see the patient and formed the basis of the TEP discussion. This meant that the doctor who conducted the TEP discussion had the most senior available opinion on what was good patient care. 
Measures:

Secondary Drivers

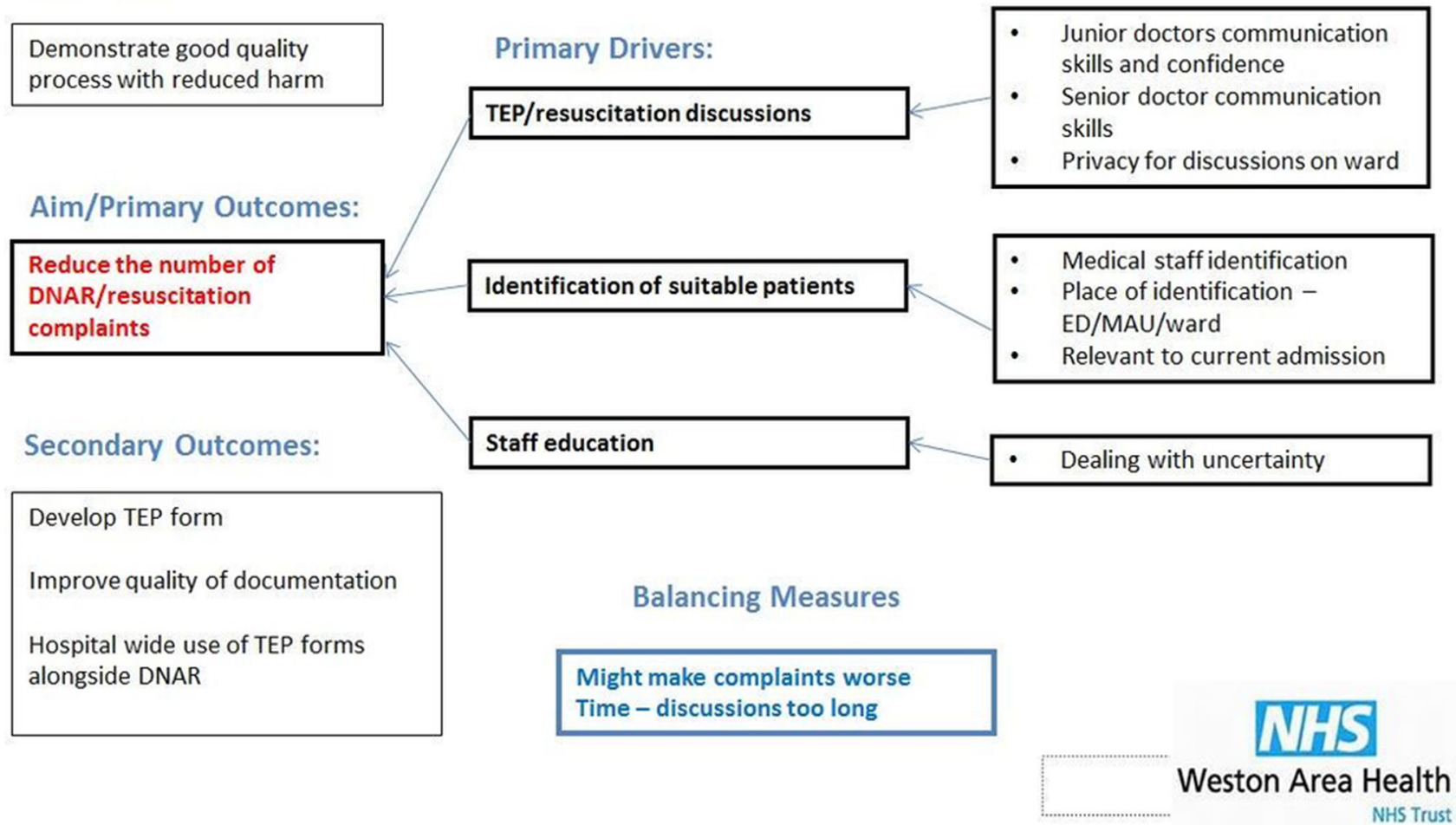

Figure 1 Driver diagram. ED, emergency department; MAU, Medical Admission Unit; TEP, treatment escalation plan.

2. Capacity assessment followed by recording of the TEP discussion with the patient and family. The discussion included decisions regarding noninvasive interventions, options concerning levels of appropriate medical and surgical interventions, artificial feeding and cardiopulmonary resuscitation treatment at the end. It was felt that a graded form of discussion was more likely to be conducted in a sequential and sensitive fashion.

3. Page 2 of the form was bordered red and was the final outcome of the discussion, including DNACPR decisions, countersigned by the consultant. A copy of the completed form was given to the patient and family where desired.

Changes were made to the initial form: the date of admission was included, 'high care' was removed as an option in the ceiling of care due to ward changes, removal of tick boxes which were poorly read and filled out, and prompts were included into the new forms to help aid the discussions. As mentioned above, we monitored these on a regular basis, making note of the positives and negatives, gaining feedback from colleagues, and installed all this information together and created the final version, which we rolled out into all the wards.

\section{STRATEGY}

We met weekly as a group to discuss the improvements or data we had gathered the previous week to suggest changes and how to measure them. Meeting weekly allowed us to make small, incremental changes in the design of the form and its roll-out across initially the medical wards followed by the surgical wards. We used the methodology of 'Adopt, Adapt, Abandon' as the basis for discussing results, with further formulation of theories which were tested in the forthcoming week.

Overall we carried out 11 PDSA improvement cycles over the course of the year August 2015 to August 2016.

These involved collecting numbers of TEP forms, changing the form itself to improve the quality, collecting feedback from our colleagues, patients and relatives, and enlisting the help of junior doctors across all the wards in the hospital to gather support for our project, increasing the sense of ownership.

Examples of PDSA cycles are given in figure 2.

Our first PDSA cycle on the care of the elderly ward was to count the number of patients with DNACPR and TEP forms. Many patients had DNACPR forms but had not had TEP discussions. We added a column onto the ward handover sheet, allowing us to see quickly which patients had DNACPR but had not had a TEP discussion. The increase in patients who had completed both in a 28-bedded ward was $4 / 28$ to $24 / 28$. This increase in TEP numbers was maintained as a consequence of this change.

Our fourth PDSA cycle added the date of admission, removing the tick boxes on the front of the form relating to advanced care planning decisions that were never filled in, and adding prompts for both the discussion and treatment plan boxes. The latter change we hoped would improve quality of documentation, as well as assisting in 


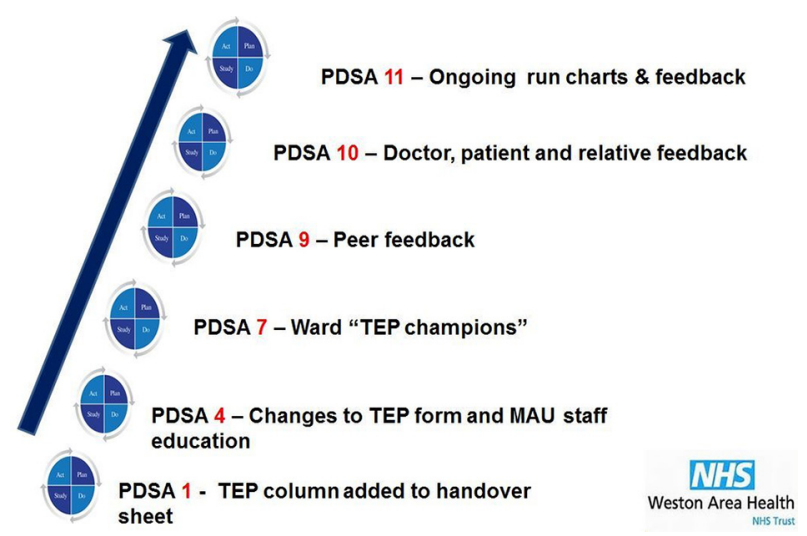

Figure 2 Plan-do-study-act (PDSA) cycle highlights. MAU, Medical Admission Unit; TEP, treatment escalation plan.

communication. We presented our TEP changes at the junior doctor teaching, and in addition went to talk to all doctors on the Medical Admission Unit about the TEP form changes to educate them on the changes. As a group we created a scale on which to judge the quality of the discussion documentation and of the treatment plan documentation. We also measured how much of the form was filled in. The quality of the discussion and treatment plan improved with the new form, so we adopted the changes.

With a new, improved form, our next focus was on how to roll out the TEP form on a large scale to all the wards. We decided on individual ownership as an important principle. To track the progression and uptake of the new forms, we counted the number of patients with DNACPR with/without TEPs across initially all the medical wards. With this in mind we presented our data about the new TEP forms to our colleagues and asked for 'TEP Champions' for each ward. With volunteers, we then went round the individual wards, explaining the changes and showing the 'TEP Champions' the spreadsheet on the intranet where they could record data they collected.

We continued to monitor the quality of performance of each section of the form and found the quality of discussion had improved, but the treatment plan quality had slightly decreased. We went back to our colleagues to gather informal feedback on whether there were any more prompts that could be helpful.

We finally surveyed the junior doctors, again raising the profile of our project in the hospital and among our colleagues, and gathering important information through white space questions, on barriers to implementation and what we could work on in the future.

\section{RESULTS}

The primary aim of this project was to reduce the number of DNACPR/resuscitation complaints as no successful intervention thus far had resulted in a reduction in resuscitation complaints. Analysis of patient liaison services data showed a significant reduction in complaints regarding resuscitation. DNACPR complaints were reduced to zero during the final six months of the project, and there were no complaints regarding the use of TEP since the project commenced (see figure 3 ).

Our secondary outcomes were to develop the form to increased doctor and patient satisfaction; improving TEP discussions and documentations and finally to introduce the TEP form across all patients with DNACPR in place.

Qualitative feedback demonstrated increased patient/ relative and junior doctor satisfaction. Patients and relatives felt it was 'useful to have copy of final plan to look at and read', 'felt involved in treatment planning' and 'felt better having a conversation with a person and the opportunity to ask questions'.

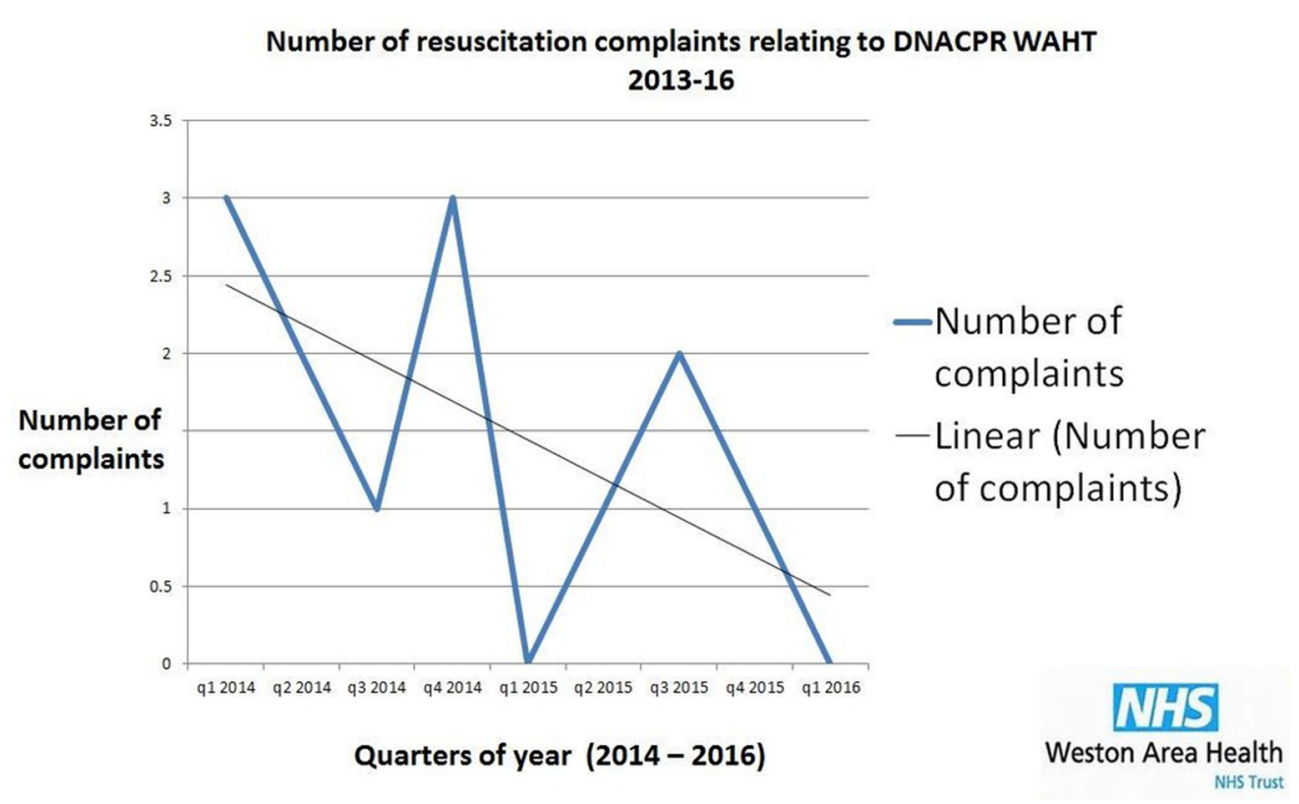

Figure 3 DNA-related complaints. 
TEP forms assist me in making decisions for deteriorating patients whilst I am on call

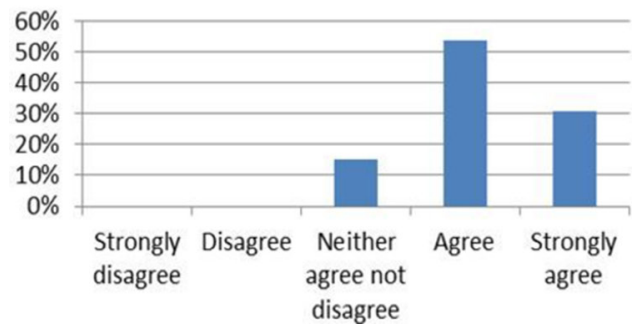

The TEP process is an improvement on the DNAR system

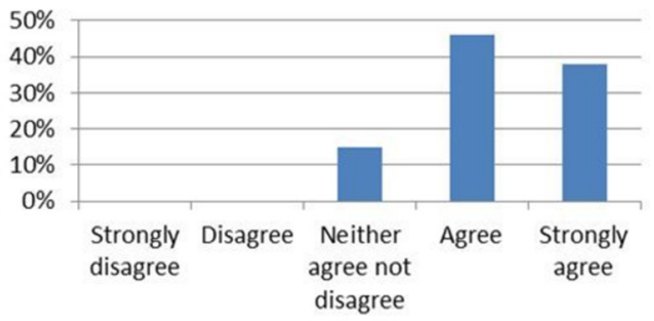

Figure 4 Junior doctor feedback. TEP, treatment escalation plan.

Of the junior doctors surveyed $85 \%$ felt that 'TEP forms assist me in discussing ceiling of care and treatment plans' and $85 \%$ felt 'TEP forms assist me in making decisions for deteriorating patients whilst I am on call'. Also, $83 \%$ of junior doctors agreed that the TEP process is an improvement on the DNACPR system. Most doctors surveyed agreed the TEP form was easy to use (77\%) or didn't agree or disagree (3\%). Fifteen per cent of junior doctors felt TEP discussions took too long (see figure 4).

We demonstrated an improvement of TEP discussion and documentation through developing the form and to include discussion and treatment plan prompts. Over two PDSA cycles sampling 10 forms at a time, we saw the scores improve. The old forms had an average quality of discussion of 1.1 and an average quality of treatment plan of 1.4. The quality of discussions improved over the PDSA cycles from 1.6 on cycle 1 to 2.4 on cycle 2 . The quality of treatment plans also improved overall, from 2.3 on cycle 1 , though then fell slightly to 2.1 on cycle 2 (see figure 5 ).

Our run charts across the medical wards demonstrated an increase in the number of TEP forms. We showed an astronomical increase in the number of TEP forms on the 28-bedded care of the elderly ward from 4/28 to 24/28 when a column was added to the ward round sheet (see figure 6). This increase in TEP numbers was maintained over the next few weeks of data collection. In addition, TEP form numbers increased over the medical wards, particularly when one of the team was a junior working on one of the wards. We feel that the user-friendly and clear layout of the final form helped to achieve all of these outcomes (figure 7).

\section{Lessons and limitations}

We were able to demonstrate that, while discussing ceiling of care is an emotive subject, it is a conversation people value. Change among health professionals is only successful if it is valued and felt to be relevant to their everyday practice. Engaging the doctors was not always straightforward, but was paramount to the reduction of DNACPR complaints.

Despite our colleagues believing that the TEP form was a helpful guide to lead advance care planning discussions, $15 \%$ still felt that this took too long, The increased quality of communication with patients and the subsequent decline in DNACPR-related complaints is a strong indication that spending that time to fully address ceiling of

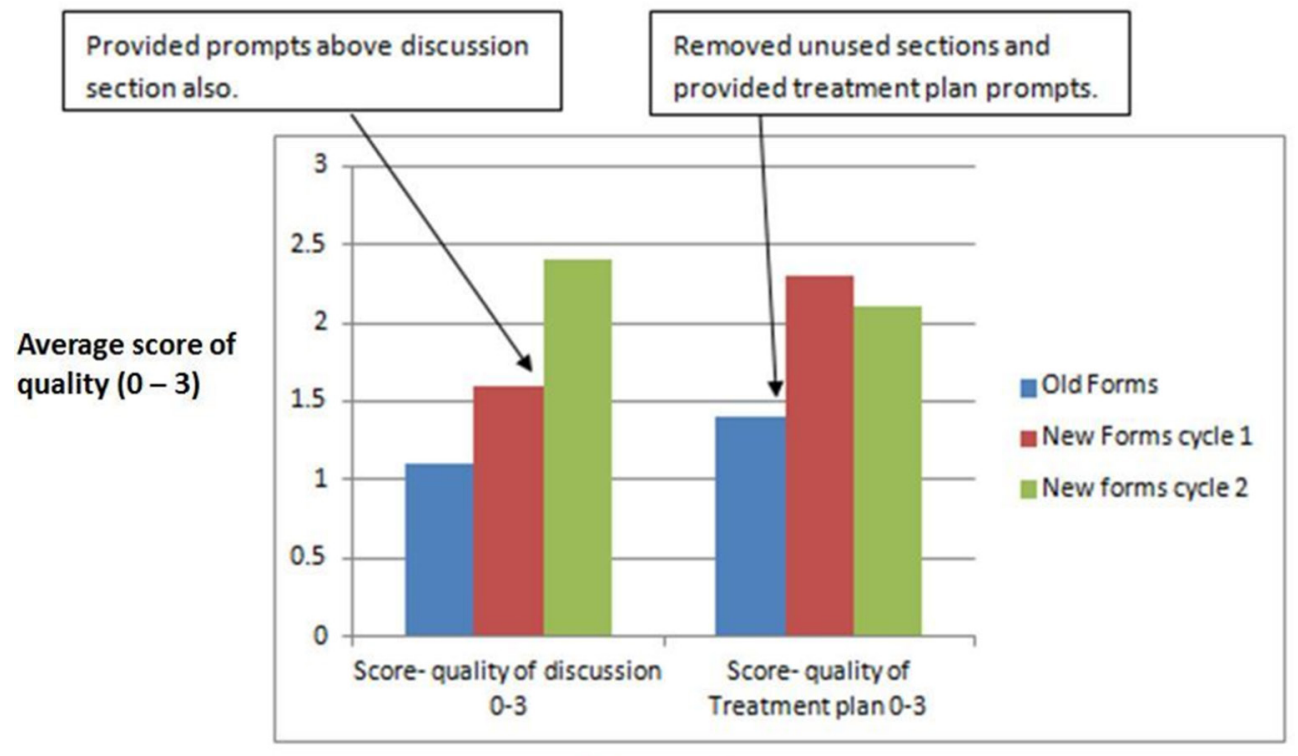

PDSA cycles 4-6

Figure 5 Improvement in treatment escalation plan from quality. PDSA, plan-do-study-act. 


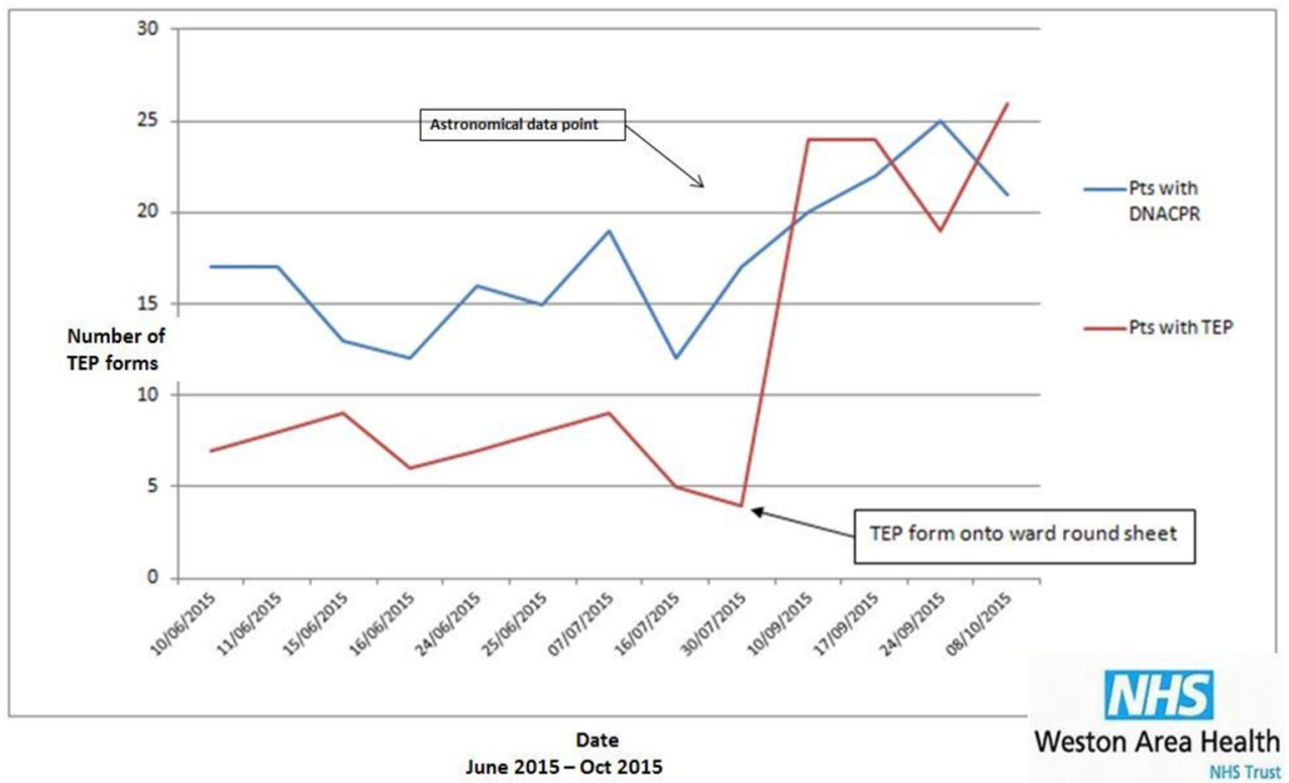

Figure 6 Run chart plan-do-study-act 1 care of the elderly ward. TEP, treatment escalation plan.

care is valuable time spent. Towards the end of our study period, we focused more on the quality of communication and discussion content. It would now be beneficial to reassess the number of patients with TEP forms in place to ensure that uptake remains good across all specialties.

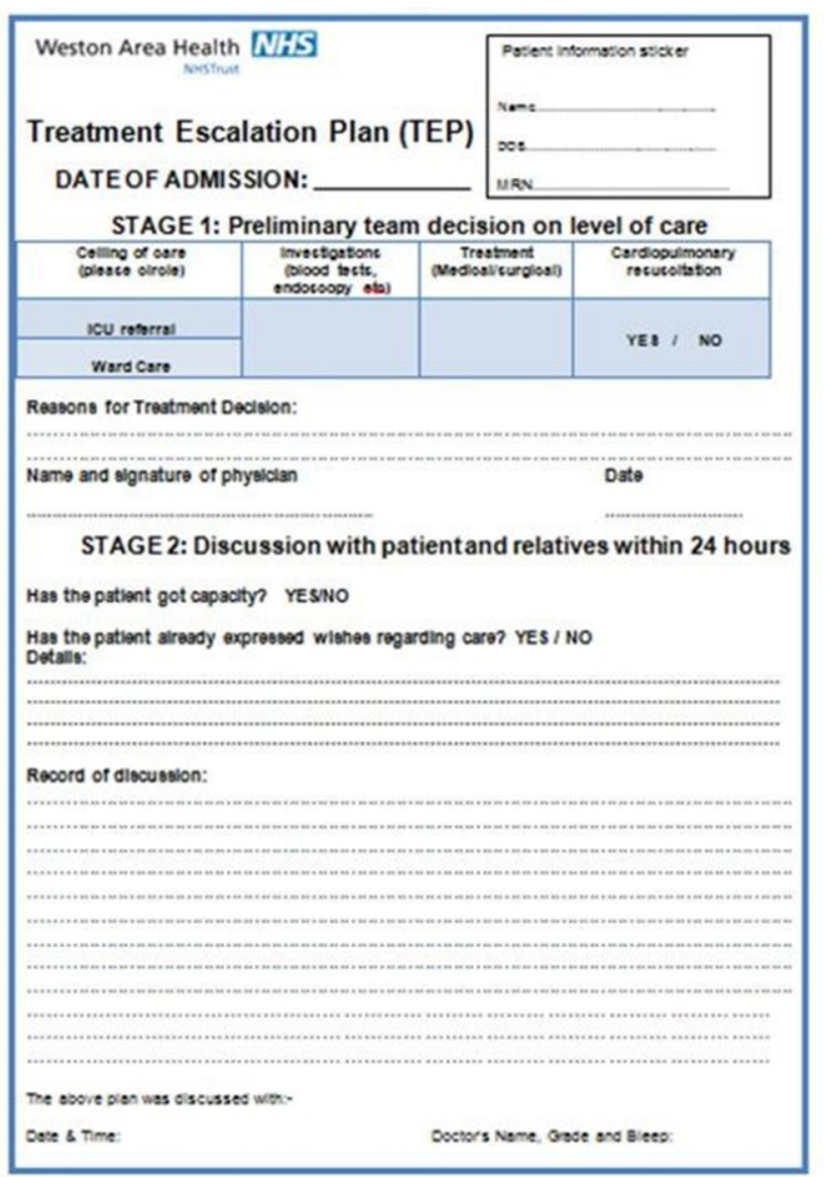

The TEP forms were initially rolled out over the care of the elderly ward, medical assessment unit and general surgical wards. We found that if a member of the TEP study team were working on those units, the uptake of TEP increased. As DNACPR complaints arise from the

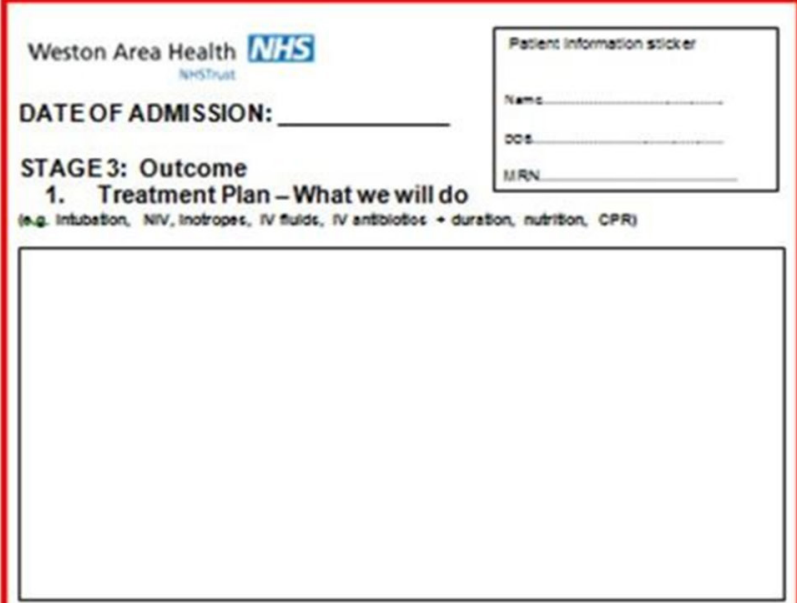

2. Agreed ceiling of care

\begin{tabular}{|c|c|c|c|}
\hline 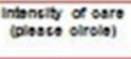 & 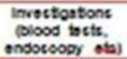 & $\begin{array}{c}\text { Troatinant } \\
\text { Modiogliverolioge? }\end{array}$ & $\begin{array}{l}\text { Cardiopulmonary } \\
\text { retureitation }\end{array}$ \\
\hline ICU rotorrs! & & & \multirow{2}{*}{ YEa, No } \\
\hline wara caro & & & \\
\hline
\end{tabular}

Consultant signature verifying discussion and decision

DATE .

COPYOF FORM GIVEN TO PATIENT/RELATIVE: YESINO

STAGE 4: Is advanced care planning on discharge appropriate?

Yes / No

Figure 7 Final treatment escalation plan form. 
whole hospital, we needed a way of ensuring TEP discussions were a priority throughout. We tackled this in two ways; asking for volunteer TEP 'Champions' based on every ward and second, ensuring TEP forms were physically available in all areas of the hospital including emergency department. Highlighting the importance that having ceiling of care discussions is everyone's responsibility and having a named 'Champion' maintained the number of TEP forms used.

If we were to repeat this project again, we would ensure TEP 'Champions' were in place from the start. This would really drive the project from the grassroots up and not rely quite so much on the project team members being present on the wards. Regular feedback was sought from all levels of the medical team, but holding regular focus groups with the different specialties would have been beneficial.

Complaints regarding DNACPR have been significantly reduced during the time period of implementing the TEP form. Together with an increase in patient, relative and doctor satisfaction, we were able to show a lasting effect of the intervention.

Junior doctors move jobs every 4-6 months and hospitals every year at a minimum. In order to keep this project running and developing, this year's team handed over to the new intake of F1 doctors. Quality improvement projects are designed to evolve so we hope that this project continues to be sustainable and achieve its aims.

\section{CONCLUSION}

This project has demonstrated how changing a poor quality form can make a significant impact. Our main aim of reducing DNACPR complaints was achieved. We showed improved communication as assessed by feedback from patients, relatives and doctors, as well as an improvement in the quality of documentation. Junior doctor confidence in leading advanced care planning discussions increased. This project also highlighted the value of junior-led quality improvement projects.

Current literature demonstrates that DNACPR forms are limited in their scope, focus on withholding treatments and do not explore what can be offered. ${ }^{4}$ Patients and relatives feel communication surrounding ceiling of care discussions has been poor and doctors felt unprepared to have them. ${ }^{4-6}$ The introduction of TEP forms in other centres improved documentation, decreased uncertainty about ceilings of care and reduced doctor anxiety. ${ }^{411}$ Our project extended this further and aimed to tackle the larger issue of DNACPR complaints. Quality improvement methodology has not been used to develop the many forms used in the National Health Service. We have shown that by using this methodology an intervention can be produced that has dramatic effects. Long term, we hope this leads to gold standard communication and documentation of TEPs and an environment of openness with our patients.

Acknowledgements The team would like to thank the doctors involved with the TEP project in the preceding years for their work in initiating TEP forms in the Trust and allowing them to continue with this quality improvement project. With thanks to Susanna Mellor, Sophie Russell, Harriet Pudge, Prashanth Mamilla and Tariq Memon.

Contributors Study design, data collection, analysis and write up performed by ES, LM, SO and LW. Study supervised by JA.

Competing interests None declared.

Ethics approval None.

Provenance and peer review Not commissioned; externally peer reviewed.

Open Access This is an Open Access article distributed in accordance with the Creative Commons Attribution Non Commercial (CC BY-NC 4.0) license, which permits others to distribute, remix, adapt, build upon this work non-commercially, and license their derivative works on different terms, provided the original work is properly cited and the use is non-commercial. See: http://creativecommons.org/ licenses/by-nc/4.0/

(C) Published by the BMJ Publishing Group Limited. For permission to use (where not already granted under a licence) please go to http://www.bmj.com/company/ products-services/rights-and-licensing/

\section{REFERENCES}

1. National End of Life Care Partnership. Ambitions for palliative and end of life care, 2015. http://endoflifecareambitions.org.uk

2. Findlay $\mathrm{G}$, Shotton $\mathrm{H}$, Kelly $\mathrm{K}$, et al. Time to intervene? A review of patients who underwent cardiopulmonary resuscitation as a result of an in-hospital cardiorespiratory arrest: A report by the National Confidential Enquiry into Patient Outcome and Death, 2012.

3. Fritz Z, Fuld J. Ethical issues surrounding do not attempt resuscitation orders: decisions, discussions and deleterious effects. $J$ Med Ethics 2010;36:593-7.

4. Obolensky L, Clark T, Matthew G, et al. A patient and relative centred evaluation of treatment escalation plans: a replacement for the donot-resuscitate process. J Med Ethics 2010;36:518-20.

5. Morgan R, King D, Prajapati C, et al. Views of elderly patients and their relatives on cardiopulmonary resuscitation. BMJ 1994;308:1677-8.

6. Hill ME, MacQuillan G, Forsyth M, et al. Cardiopulmonary resuscitation: who makes the decision? BMJ 1994;308:1677.

7. Wilson J. To what extent should older patients be included in decisions regarding their resuscitation status? J Med Ethics 2008;34:353-6.

8. Löfmark R, Nilstun T. Do?not?resuscitate orders-should the patient be informed? J Intern Med 1997;241:421-5.

9. Sivakumar R, Knight J, Devlin C, et al. Communicating information on cardiopulmonary resuscitation to hospitalised patients. J Med Ethics 2004;30:311-2.

10. Decisions relating to cardiopulmonary resuscitation: a joint statement from the british medical association, the resuscitation council (UK) and the royal college of nursing. J Med Ethics 2001;27:310-6.

11. Dahill M, Powter L, Garland L, et al. Improving documentation of treatment escalation decisions in acute care. BMJ Qual Improv Rep 2013;2:u200617.w1077-w1077 . 